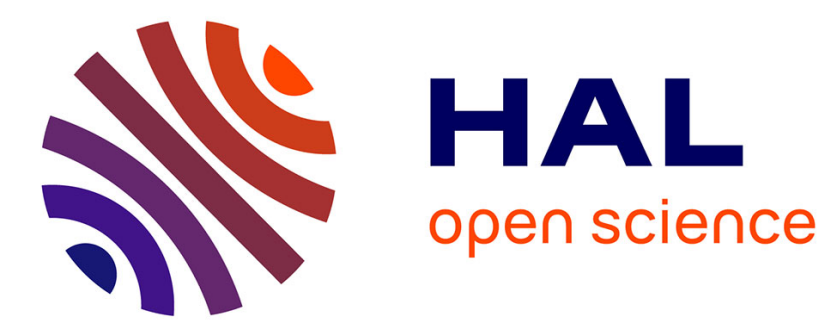

\title{
Detection and emission of radiation by arrays of junctions \\ T.D. Clark
}

\section{To cite this version:}

T.D. Clark. Detection and emission of radiation by arrays of junctions. Revue de Physique Appliquée, 1974, 9 (1), pp.207-216. 10.1051/rphysap:0197400901020700 . jpa-00243741

\section{HAL Id: jpa-00243741 \\ https://hal.science/jpa-00243741}

Submitted on 1 Jan 1974

HAL is a multi-disciplinary open access archive for the deposit and dissemination of scientific research documents, whether they are published or not. The documents may come from teaching and research institutions in France or abroad, or from public or private research centers.
L'archive ouverte pluridisciplinaire HAL, est destinée au dépôt et à la diffusion de documents scientifiques de niveau recherche, publiés ou non, émanant des établissements d'enseignement et de recherche français ou étrangers, des laboratoires publics ou privés. 


\title{
DETECTION AND EMISSION OF RADIATION BY ARRAYS OF JUNCTIONS (*)
}

\author{
T. D. CLARK
}

School of Applied and Engineering Physics, and Laboratory of Atomic and Solid State Physics Cornell University, Ithaca, New York, USA 14850

\begin{abstract}
Résumé. - Nous discutons les interactions entre un ensemble plan de jonctions Josephson et un champ électromagnétique self-induit ou extérieur. Nous décrivons les données expérimentales nécessaires pour l'observation d'un état «superradiant» monochromatique.

Abstract. - We discuss the interaction of small regular planar arrays of point contact Josephson junctions with an electromagnetic field, either self-generated or externally applied. We review the experimental data obtained in terms of a single frequency, "superradiant " state for the point contact array.
\end{abstract}

1. Introduction. - In 1967 Zimmer [1] reported briefly on the interaction of microwaves with partially agglomerated films of superconducting tin. One or two of his tin samples showed parametric amplification. Zimmer suggested that one or more Josephson weak links, produced by chance where the agglomerates of tin touched one another, were responsible for this nonlinear property. To us, Zimmer's data appeared to show that under the right circumstances large numbers of Josephson weak links could be coupled together coherently. The " granular " [2] tin films used by Zimmer were presumably forced into overall synchronization [3], [4] by the applied microwave field.

Data derived from the study of agglomerated superconducting films are necessarily difficult to interpret. A more straightforward approach is to examine the properties of perfectly regular arrays of weak links, connected by bulk superconducting paths. We proposed what in some respects was a model granular superconductor [5] by making planar arrays of uniform diameter superconducting spheres. We expected that where the spheres touched (under external pressure) Josephson weak links of small cross-sectional area would be formed. The principal drawback of such arrays is their mechanical instability, at least compared with array networks utilising microbridges or tunnel oxide junctions. However, we expected planar sphere arrays to couple easily to external microwave fields, since touching spheres should serve as a relatively efficient structure for launching a TE wave from

(*) Research supported in part by the US Office of Naval Research Grant \# N000-67-A-0077-0016. a low impedance Josephson point contact junction [6], [7]. Figure 1 shows the typical arrangement of a superconducting sphere array.

In the kind of point contact array shown in figure 1 it is highly unlikely that the normal state conductances of the contact regions should all be the same. If the array is fed from a single voltage source, through electrodes at each end of the array, one of two possible states seems possible. On the one hand, the array can be in what could be called an "uncorrelated " state, in which each junction in the array has its own unique dc voltage $V$ and interacts with its own photon field of frequency $\omega=2 \mathrm{e} V / \hbar$. On the other hand,

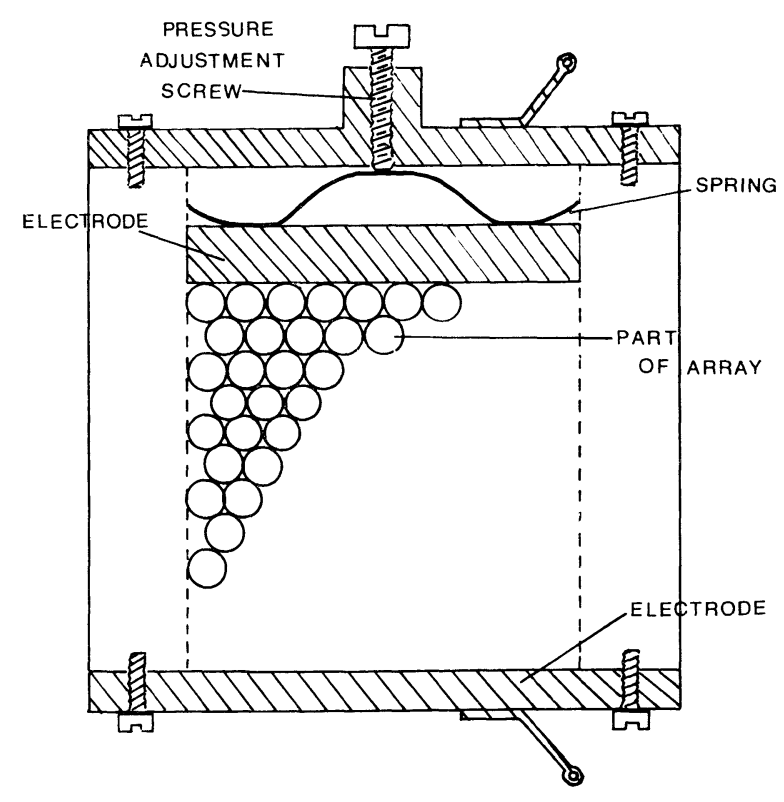

FIG. 1. - Schematic of a two-dimensional array. 
the array can be in a "correlated " state - oscillating at a single frequency - in which all the dc voltages are equal to the Josephson value $V_{0}=\hbar \omega_{0} / 2 \mathrm{e}$, or some multiple or submultiple of this value. In this « single " frequency condition the junctions interact with a common radiation field.

D. R. Tilley [8] has examined these two possibilities. For simplicity he considered the interaction of a linear array of $M$ weak links, connected by bulk superconducting paths, and fed from a single voltage source. He assumed that self-synchronization [9] of the array can occur through the interaction of the array with an external electromagnetic cavity - each weak link in the array being coupled to the common radiation field in the cavity. Obviously this process of self-synchronization must be strongest near to a cavity resonance. It will act to make the voltage drops across all the weak links equal. The amount of voltage pulling will depend on the strength of the radiation fields and the degree of feedback from the cavity to the array.

According to Tilley [8] the photon density $n$ in the cavity with the array operating in the correlated state is given by

$$
n=\frac{G^{2}}{(\Omega-\omega)^{2}+\Gamma^{2}} .
$$

Here $G$ describes the coupling of the array to the common radiation field; $G$ is given by the expression

$$
\begin{aligned}
G^{2} & =\sum_{m} g_{m}^{2}+ \\
& +\sum_{m \neq m^{\prime}} g_{m} g_{m^{\prime}} \cos \left\{\left(\varphi_{m}-\alpha_{m}\right)-\left(\varphi_{m^{\prime}}-\alpha_{m^{\prime}}\right)\right\},
\end{aligned}
$$

where $\omega / 2 \mathrm{e}$ is the dc bias across any weak link (taking $\hbar=1$ ), $\Omega$ is the cavity resonance frequency, $\Gamma$ is the cavity width, $\varphi_{m^{\prime}}$ is the relative pair phase, $\alpha_{m^{\prime}}$ is the radiation phase, $g_{m}$ is the coupling constant to the radiation field at each weak link ( $m=1$ to $M$ ), and $\varphi_{m}-\alpha_{m}$ is determined self-consistently by

$$
\sin \left(\varphi_{m}-\alpha_{m}\right)=\frac{J-S_{m}(\omega)}{2 g_{m} n^{1 / 2}}
$$

Here $J$ is the current passing through each weak link and $S_{m}$ represents the quasiparticle background current.

Making all the weak links identical (i. e. with $S_{m}=S, g_{m}=g$ and all the $\left(\varphi_{m}-\alpha_{m}\right)$ terms the same) considerably simplifies matters. In this case the Hamiltonian for the system is given by

$$
\begin{aligned}
H^{\prime}= & \frac{-M^{2} g^{2}(\Omega-\omega)}{(\Omega-\omega)^{2}}+\Gamma^{2}- \\
& -\frac{2 M^{2} g^{2} \Gamma}{(\Omega-\omega)^{2}+\Gamma^{2}} \arctan \frac{\Gamma}{(\Omega-\delta)}
\end{aligned}
$$

with

$$
G^{2}=M^{2} g^{2}
$$

and

$$
n=\frac{M^{2} g^{2}}{(\Omega-\omega)^{2}}+\Gamma^{2} .
$$

$n \propto M^{2}$ is the superradiant property [10], [11] of $M$ coherent radiations and in this state $\left|H^{\prime}\right| \propto M^{2}$, compared with $\left|H^{\prime}\right| \propto M$ for the uncorrelated state. Thus it appears that the correlated state is the favored one for arrays with reasonably large $M$, even though the normal state conductances (through $S_{m}$ ) of the weak links comprising the array may vary considerably. Large differences in normal state properties and coupling constants $\left(g_{m}\right)$ should prove tolerable for very large $M$ [1], [12], [13] (say $\gtrsim 10^{6}$ ).

In such a superradiant state the array should behave essentially as a single weak link, with a coupling constant to the common radiation field of $G^{2}=M^{2} g^{2}$, and an $I$ versus $V$ characteristic that is scaled up in voltage by the number of weak links $M$, across the array between the electrodes. Self-induced steps [9] in $I$ versus $V$ caused by radiative feedback from the cavity to the array should occur at a voltage $V_{\mathrm{c}}=M \hbar \omega_{\mathrm{c}} / 2 \mathrm{e}$, where $\omega_{\mathrm{c}}$ is the cavity resonance frequency. Furthermore, constant voltage current steps [14] due to the interaction of the array with an external microwave field (frequency $\omega_{\mathrm{rf}}$ ) should occur at voltage intervals of $M \times \hbar \omega_{\text {rf }} / 2$ e. For real arrays, with variations in the properties of the weak links, the $M$ scaling factor will still occur, but the interference terms in eq. (2) will reduce both $G^{2}$ and $n$.

On the above model we would not expect weak link arrays to have any unusual dc $(\omega=0)$ properties. However Rosenblatt et al. [15], [16] have proposed that as an exact analogue of the paramagnetic-ferromagnetic transition in the Ising model, a coherence transition to a truly resistanceless state (with no phaseslip) occurs at a certain temperature below the critical temperature of the bulk grains making up the array. Whether or not this state truly exists the approach is an interesting one.

In the following sections we demonstrate that pointcontact arrays do indeed have intriguing ac Josephson properties. Apparently they can enter a single frequency state in which all the junctions in the array oscillate at the same frequency and in phase with one another.

2. dc Properties. - The dc properties of arrays of tin spheres correspond closely to those predicted for single Josephson weak links. Thus all tin arrays measured by us (two- and three-dimensional) showed a resistance-versus-temperature transition starting at the bulk critical temperature $T_{\mathrm{c}}$ for tin $(3.72 \mathrm{~K})$. Rouding-off of the resistance transition, both above [17] and below [5] $T_{\mathrm{c}}$ (bulk) was attributed to the effect of fluctuations in the contact regions. Criti- 

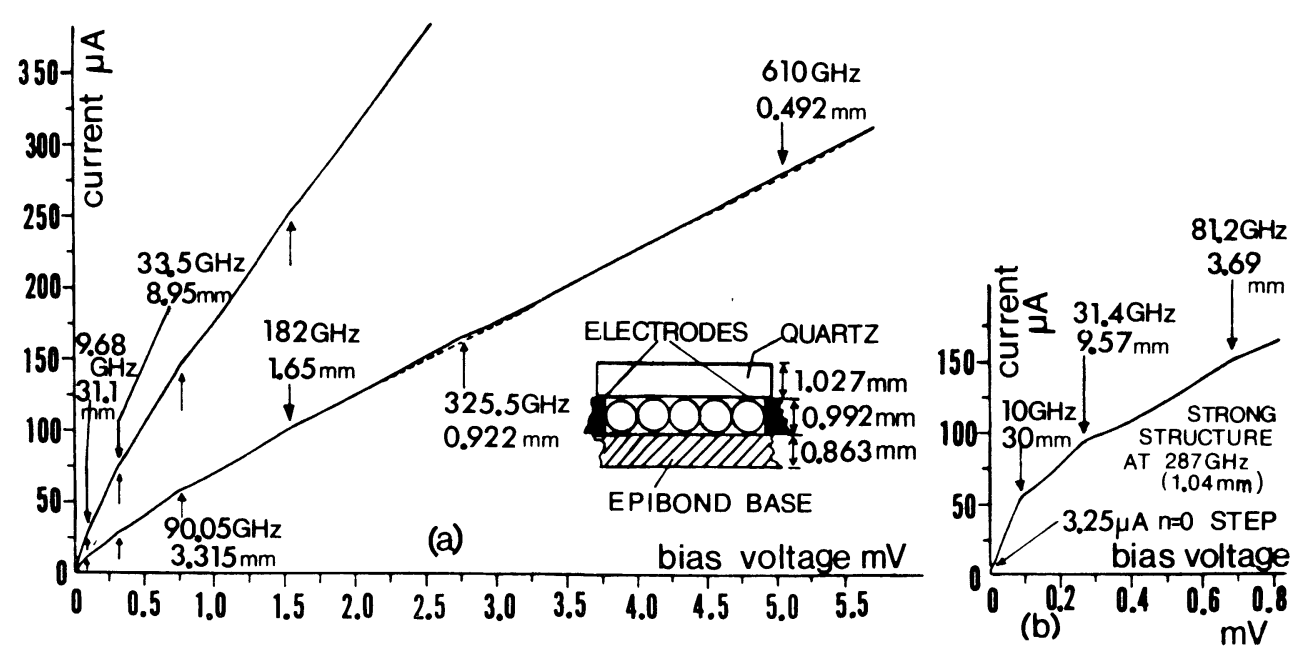

FIG. 2. $-I$ versus $V$ curves for two $5 \times 5$ square arrays of $1 \mathrm{~mm} \mathrm{Nb}$ spheres. Inset in $(a)$ is a sectional view of the array in its holder. Frequencies and wavelengths listed in $(a)$ and $(b)$ are calculated on the single frequency argument. Mean sphere diameter for array (a) $1.003 \mathrm{~mm}$; for array $(b) 1.029 \mathrm{~mm}$.

cal current versus temperature curves for tin arrays followed the theoretical calculation of Ambegaokar and Baratoff [18] quite closely [19] except very close to $T_{\mathrm{c}}$. In the normal state $I$ versus $V$ is linear. However, immediately below $T_{\mathrm{c}}$ (bulk) excess currents appear - the current-voltage curve becomes convex upwards. With regard to these excess currents, which increase as the temperature decreases below $T_{\mathrm{c}}$, the $I$ versus $V$ curve appears to be scaled up in voltage (compared to a single contact) by the order of the number of contacts across the array between the electrodes [19].

The critical current of planar arrays of tin spheres varied with magnetic field, this being applied normal to the plane of the array. From the slow « Fraunhofer $"$ period due to the magnetic flux threading the contact regions, we estimate the contacts $\lesssim 0.4 \mu \mathrm{m}^{2}$ in area, which seems about the right size for weak links.

3. ac Properties : self-synchronized state. - We have considered how an array of radiating point contact junctions might interact with an external resonant cavity. Internal modes of oscillation may also be possible, but coupling to an external cavity is obviously easier to consider. In each case the resonant structure acts to enhance radiative feedback and hence selfsynchronization of the array.

It is well known [9] that the $I$ versus $V$ curves of single point contact junctions situated in microwave resonant cavities contain current peaks at voltages (or frequencies) corresponding to the cavity resonance frequencies. When the junction is current-fed these show up as current steps. Weaker junction-cavity coupling, or lower effective cavity « $Q$ », smears out these steps considerably. Similar, "self-induced» structure is seen in the $I$ versus $V$ curves of superconducting sphere arrays. Weak structure of this type was observed even without the benefit of an external resonant cavity, as shown in figure 2 . The $5 \times 5$ square array of $1 \mathrm{~mm} \mathrm{Nb}$ spheres, mounted in a holder of Epibond and fused quartz was situated inside an $\mathrm{X}$-band waveguide. The most striking features of this $I$ versus $V$ curve are the apparently harmonically and subharmonically related series of bumps or shouldered peaks. Probably this structure arises from the weak interaction of the whole array, either internally or with some low $Q$ external, for example, the array holder itself. Most of the arrays studied showed this kind of behavior. External cavities increased in amplitude of the self-induced structure very considerably.

With the help of externally tunable resonant cavities, significant information concerning the radiant state of planar point contact arrays could be obtained. For most of these experiments the cavity comprised an external Fabry-Pérot resonator with the array of $1 \mathrm{~mm}$ tin spheres placed centrally between the mirrors of the Fabry-Pérot, as illustrated in figure 3. Typical

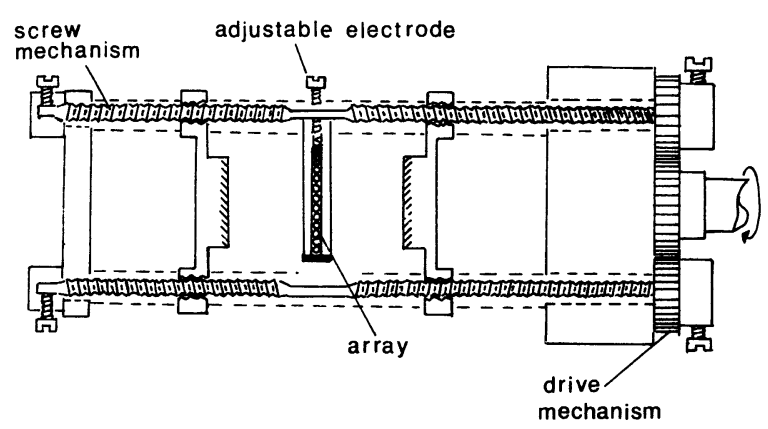

FIG. 3. - Sectional drawing of the Fabry-Pérot system.

harmonic/subharmonic structure in the array characteristic is shown in figure 4. Here, the first derivative $\mathrm{d} I / \mathrm{d} V$ versus $V$ is taken for clarity (and sensitivity). We assumed that the shouldered peaks in $I$ versus $V$ 
corresponding to the minima in $\mathrm{d} I / \mathrm{d} V$ versus $V$ arose out of the non-linear self-coupling of the array, via the external resonator. We also assumed that with the array voltage-biased exactly at the minima in $\mathrm{d} I / \mathrm{d} V$ versus $V$, the array would be on resonance.

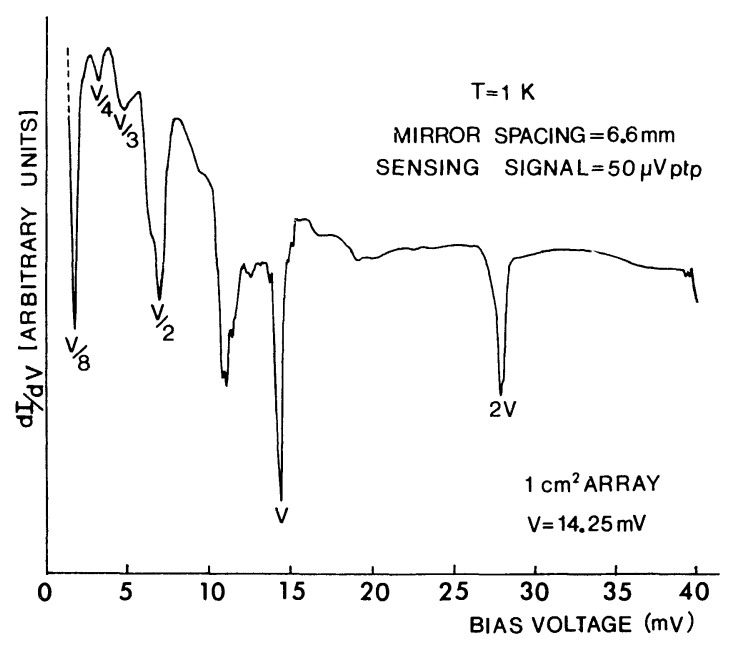

Fig. 4. $-\mathrm{d} I / \mathrm{d} V$ versus $V$ curve for a planar array of $1 \mathrm{~mm}$ tin spheres placed centrally in a Fabry-Pérot cavity. The array was 10 spheres wide and 14 spheres deep (between the electrodes). The sharp dip in the curve at $40 \mathrm{mV}$ is the start of the $3 \mathrm{~V}$ minimum.

If the array is simply driven by the cavity and has no preferred resonant frequencies of its own, we should expect to see no periodic changes in $\mathrm{d} I / \mathrm{d} V$ as a function of mirror spacing. The array-cavity system would be at resonance for all mirror spacings and we would expect to observe a shift in the selfinduced structure toward lower voltages as the mirrors were moved apart, together with a slow decrease in $\mathrm{d} I / \mathrm{d} V)_{V=V_{\min }}$ as the mirror spacing and the $Q$ of the cavity increased [20]. However if the array does have preferred frequencies, then changing the mirror spacing will change the coupling between the array and cavity very significantly. It occurred to us that if the latter were the case, the array-cavity system would act as its own crude Fourier transform spectrometer if, at fixed bias voltage, the differential conductance $\mathrm{d} I / \mathrm{d} V$ was monitored as a function of mirror spacing. On resonance, i. e. at a minimum in $\mathrm{d} I / \mathrm{d} V$ versus $V$, the radiation emitted by the array into the cavity should be a maximum. We would expect the minimum in $\mathrm{d} I / \mathrm{d} V$ versus $V$ to become shallower as the array is moved off resonance by the change in the separation of the Fabry-Pérot mirrors.

Periodic variations in the dynamic conductance of the array, at fixed bias voltages, were indeed observed. But only in the region of the shouldered peaks in $I$ versus $V$, i. e. the minima in $\mathrm{d} I / \mathrm{d} V$ versus $V$. The results have already been discussed in earlier publications [19], [21]. Gregory and his coworkers [22] have confirmed these findings. Recently, this technique of self-detection has been used by Ulrich and Kluth [23] to observe radiation emitted by a single point contact junction situated in a resonant cavity.

Since the spacing plot is a Fourier transform of the power spectrum of the emitted radiation, Fourier transforming this plot should provide at least an approximate spectrum of the radiation emitted from the array. Figure 5 shows the result of Fourier transforming a typical interferogram. As can be seen, the transform peaks very sharply at $1.1 \mathrm{~mm}$ and $0.56 \mathrm{~mm}$, corresponding to the fundamental and second harmonic of the emitted radiation.

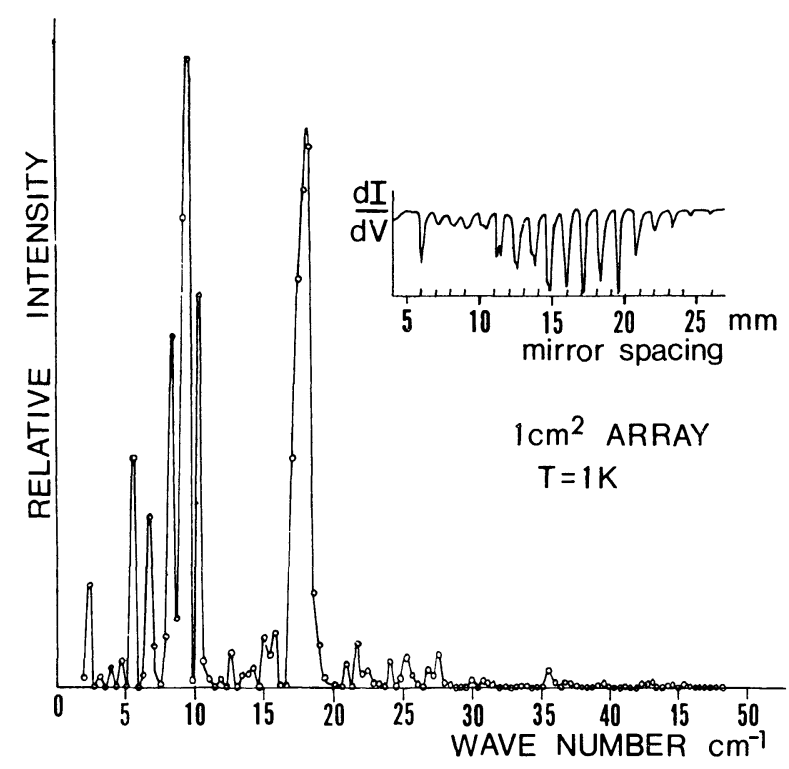

FIG. 5. - Fourier transform of array interferogram shown in inset. This transform reveals that the radiation emitted by the array peaks sharply at $1.1 \mathrm{~mm}$ and $0.56 \mathrm{~mm}$.

Generally, we found that only the fundamental and harmonic peaks varied in amplitude periodically, although Repici et al. [22] find that the subharmonic structure also shows this behavior. The dominant period in figure 5 is $1.16 \mathrm{~mm}$ which, we assume, corresponds to the wavelength of the self-detected radiation. In other arrays a dominant period, close to $1 \mathrm{~mm}$, was regularly observed. The subharmonic minima showed changes in amplitude with change of mirror spacing, but usually no periodicity was apparent. Figure 6 shows the marked changes in the selfinterferogram as the bias voltage is swept through a minimum in $\mathrm{d} I / \mathrm{d} V$ versus $V$. It is worth noting that no periodic changes in $\mathrm{d} I / \mathrm{d} V$ with mirror spacing are seen outside the minimum.

Changes in the differential resistance of the array, at fixed bias voltages, as a function of mirror spacing indicate that the greatest $V^{2}\left(1 / R_{1}-1 / R_{2}\right)$ power difference between the maxima and minima in figures 5 and 6 is $\sim 10^{-5} \mathrm{~W}$. Given the accepted radiated power levels of single point contact junctions [9] this indicates that the array is in the correlated state with the power fed into the cavity going as $M^{2}$, where $M$ is the number of junctions in the array. 


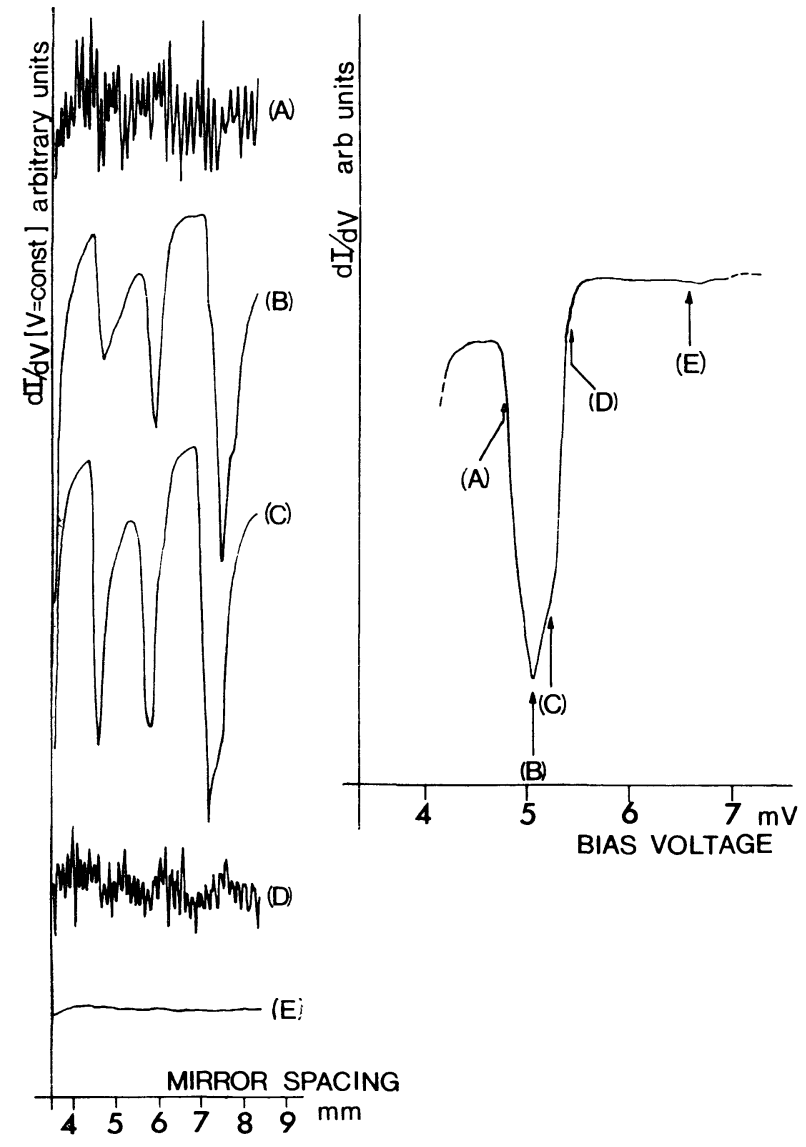

FIG. 6. - Variation of $\mathrm{d} I / \mathrm{d} V$ at fixed biased voltage with mirror spacing for a $1 \mathrm{~cm}^{2}$ array of $1 \mathrm{~mm}$ Sn spheres. Left side shows mirror scans for the various bias positions $\mathrm{A}$ to $\mathrm{E}$ on the right $\mathrm{d} I / \mathrm{d} V$ versus $V$ curve.

The antenna properties of these planar sphere arrays are probably responsible for the observed operating wavelength. The correlation energy for the array will be a maximum when the radiative feedback is optimized. For a stacked broadside array of in-phase dipoles [7] the array will have a maximum forward power gain for emission of radiation when the dipole spacing $d \sim$ half the wavelength $\lambda$ of the radiation. By the reciprocity theorem, maximum feedback from the cavity to the array will also occur when $\lambda \sim 2 d$, i. e. for $1 \mathrm{~mm}$ diameter spheres, in a close-packed array, $\lambda$ should be close to $1 \mathrm{~mm}$.

The above experimental data provide strong evidence that the array-cavity system can, under appropriate circumstances, oscillate predominantly at one frequency, at least when the array is voltage-biased into the neighborhood of the self-induced structure in $I$ versus $V$. Obviously, the Fabry-Pérot experiment offers a somewhat indirect, if nonetheless meaningful, proof of the existence of the superradiant state. Recently, however, T. Finnegan and S. Wahlsten [24] have shown by direct superheterodyne detection of the emitted radiation that two tunnel oxide Josephson junctions connected in series can enter a superradiant state. Dr. Finnegan will expand on these elegant experiments in another lecture.
4. ac Properties : driven state. - If it is possible to synchronize a complete weak link array by means of an external microwave field, constant voltage current steps should be seen in the current-voltage characteristic of the array at voltage intervals of $M \times \hbar \omega / 2 \mathrm{e}$. $M$, as before, is the number of weak links across the array, between the electrodes. Under suitable circumstances and at certain microwave power levels synchronization was indeed observed, as has already been reported [19]. Also, if the array is truly in the singlefrequency condition, and the critical current of the array is suitably adjusted, the various orders of the microwave induced current steps should change in amplitude with $v_{0}$ as would a single weak link, viz. as $J_{0}\left(2 \mathrm{e} v_{0} / \hbar \omega\right), J_{1}\left(2 \mathrm{e} v_{0} / \hbar \omega\right), J_{2}\left(2 \mathrm{e} v_{0} / \hbar \omega\right), \ldots$, etc., where $v_{0}$ is the rf voltage.

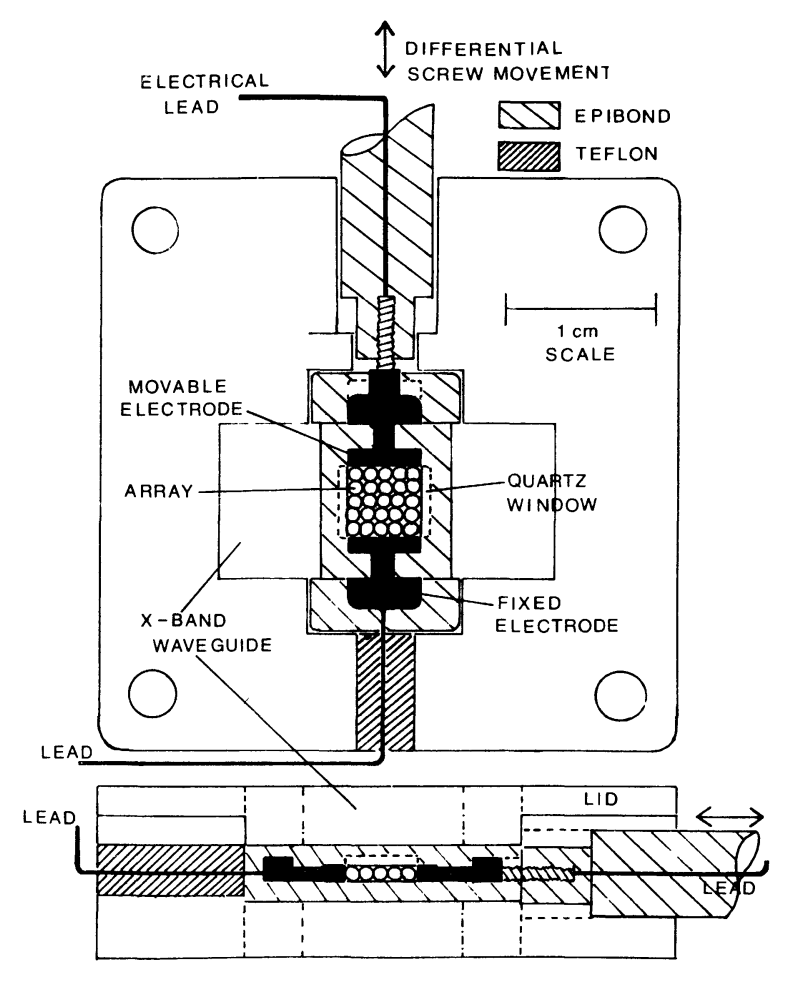

Fig. 7. - Schematic of array mounting structure and pressure adjustment system used in microwave experiments.

Figure 8 shows a set of $I$ versus $V$ curves for a $5 \times 5$ square array of $1 \mathrm{~mm} \mathrm{Nb}$ spheres (X-band waveguide arrangement shown in Fig. 7) at various levels of rf power. The steps are not truly vertical, presumably due to the effects of external noise [25], [26] on the high resistance (low critical current) array. However, the Josephson relationship, $\hbar \omega=2 \mathrm{ev}$ apparently still holds if the voltage intervals are measured from the mid-current points of the steps. On this basis, the voltage between steps as measured on the $-1 \mathrm{~dB}$ curve is $73.5 \pm 2.4 \mu \mathrm{V}$, i. e. close to $4 \times \hbar \omega / 2 \mathrm{e}(\hbar \omega / 2 \mathrm{e}=18.92 \mu \mathrm{V}$ and $v=9.15 \mathrm{GHz})$.

Since $M$, the number of possible weak link contacts across the array between the electrodes equals 4 , 


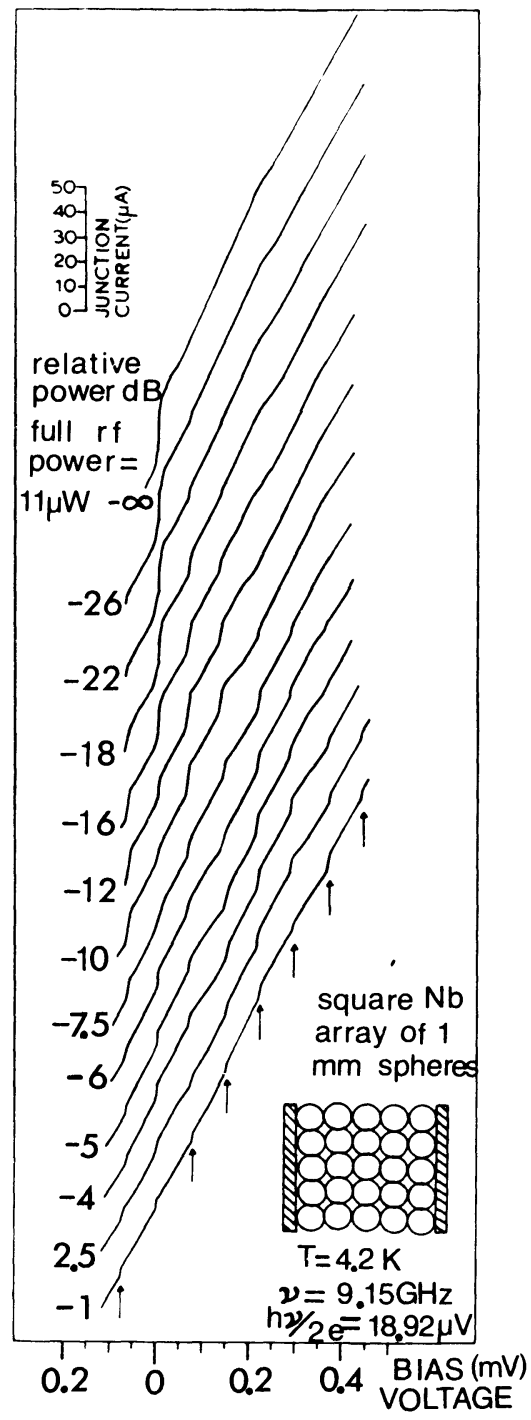

Fig. 8. - Set of $I$ versus $V$ curves for a $5 \times 5$ square array of $1 \mathrm{~mm} \mathrm{Nb}$ spheres at various levels of microwave power.

it does appear that the whole array is being forced to synchronize with the microwave field. We have discounted weak link contacts parallel to the electrodes, i. e. normal to the $E$-field of the dominant $\mathrm{H}_{10}$ (transverse electric) mode in the rectangular waveguide.

Figure 9 shows the dependence of the various step amplitudes on rf voltage. As can be seen, this array appears to show good Bessel behavior. Analogue studies [27] of simple equivalent circuits representing a single Josephson weak link driven by an external microwave field indicate that good Bessel behavior for the microwave-induced steps will occur only when the parameter [28], [29] $\left(\hbar \omega / 2\right.$ e) $R^{-1} I_{0}^{-1} \gtrsim 1$, where $\omega$ is the frequency of the microwave signal, $R$ is the parallel resistance and $I_{0}$ is the maximum zero voltage supercurrent. If a correlated array of four weak links in series (our $5 \times 5$ array) can be similarly treated in terms of lumped components, we would expect the critical parameter to become

$$
4 \times(\hbar \omega / 2 \text { e }) R^{-1} I_{0}^{-1} \text {. }
$$

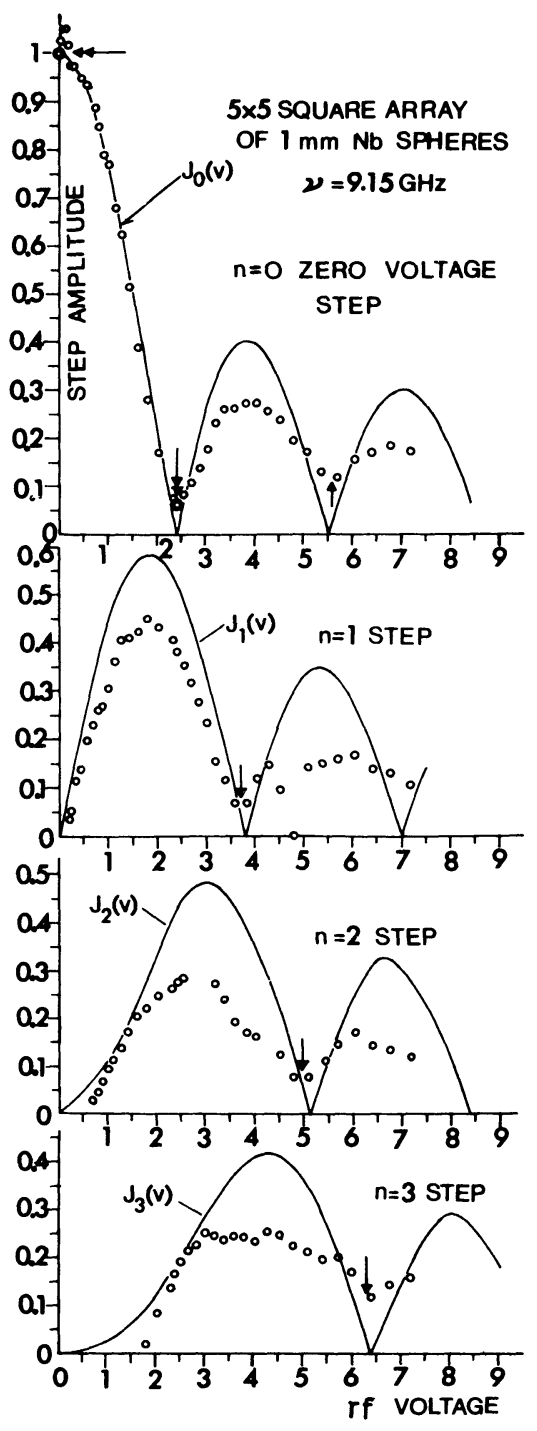

FIG. 9. - Plots of step amplitude versus $\mathrm{rf}$ voltage for the steps $n=0$ to 3. Bessel functions $J_{0}(v)$ to $J_{3}(v)$ are shown as full curves. Matching points for the four sets of data are shown ringed and marked by double arrows. The estimated positions of the minima in the experimental curves are marked by arrows.

For the data presented in figure 8 , we find that $\hbar \omega / 2 \mathrm{e}=18.92 \mu \mathrm{V}, R_{\text {normal }} \simeq 2.5$, and $I_{0}=12 \mu \mathrm{A}$, giving

$$
4 \times \frac{\hbar \omega}{2 \mathrm{e}} R^{-1} I_{0}^{-1} \simeq 2.4
$$

The zero voltage supercurrent step appears to show a small increase in amplitude $(\sim 5 \%)$ at low levels of microwave power. Small superconducting bridges often show similar behavior, the so-called Dayem (or Watt) effect [30] over a limited range of temperature.

"Correlated " steps were also seen in the $I$ versus $V$ characteristics of close-packed arrays. Once again $M=4$. Since more detail could be obtained from the derivative curve we plotted $\mathrm{d} V / \mathrm{d} I$ versus $V$ at various rf power levels. Two of these derivative curves are 
presented in figure 10. The sharp minimum at $V=0$ corresponds to the zero voltage supercurrent of the array. The voltage interval between these steps is accurately $4 \times \hbar \omega / 2 \mathrm{e}=76 \mu \mathrm{V}$ for a microwave frequency of $9.18 \mathrm{GHz}$ (equivalent to a Josephson voltage $\hbar \omega / 2 \mathrm{e}=19 \mu \mathrm{V})$. At the highest power levels ( $\sim 2 \mathrm{~mW})$ over 100 correlated steps were counted. A typical derivative curve for high input powers is shown in figure 11 .

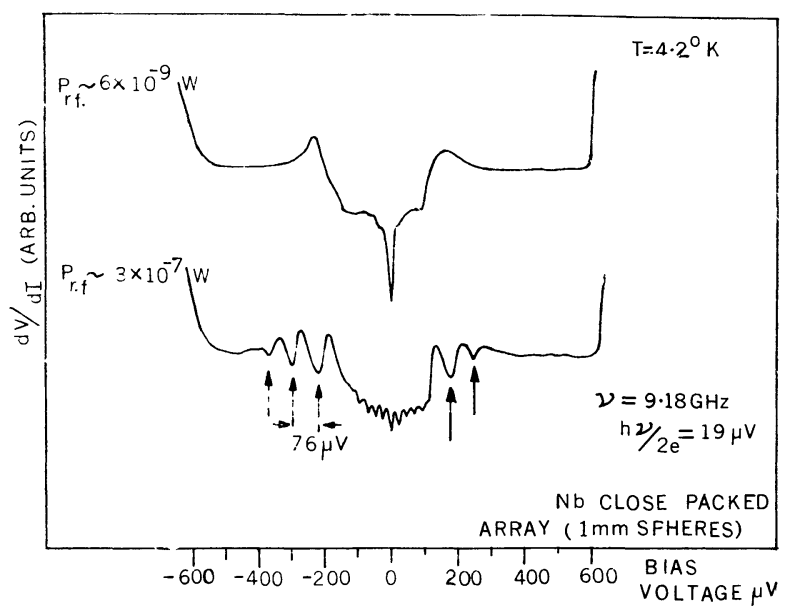

FIG. 10. - Initial build up of correlated steps at low rf power levels for a $5 \times 5$ close-packed array of $1 \mathrm{~mm} \mathrm{Nb}$ spheres.

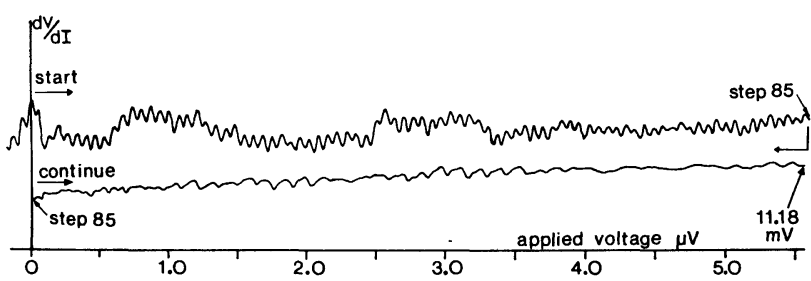

FIG. 11. $-\mathrm{d} V / \mathrm{d} I$ versus $V$ for the array of figure 10 taken to high bias voltages. Microwave input power $=2 \mathrm{~mW}$. For voltages $>1 \mathrm{mV}$ the array shows complex switching behavior between the fully correlated state $(V=4 \hbar \omega / 2 \mathrm{e})$ to the partially correlated state $(V=3 \hbar \omega / 2 \mathrm{e})$. Microwave-induced structure extends to at least $11 \mathrm{mV}$.

One noticeable feature of these curves is that, unlike those for the square array, the first $(n=1)$ correlated step is missing. This is shown very clearly in figure 12. Presumably the way the array couples to the microwave field at low power levels depends on the geometry of the array. Certain geometries (viz. the square array) may be favored over others in forming a correlated state for weak synchronizing fields.

Obviously no fully correlated steps will be observed at bias voltages $<M \hbar \omega / 2 \mathrm{e}$. However, what happens in the voltage interval $0<V<M \hbar \omega / 2$ e presumably depends on the strength of the coupling of the individual junctions to the microwave field. At low power levels only one junction appears to be active in this voltage range since the minima are spaced at regular $20 \mu \mathrm{V}$ intervals. The voltage-dependent

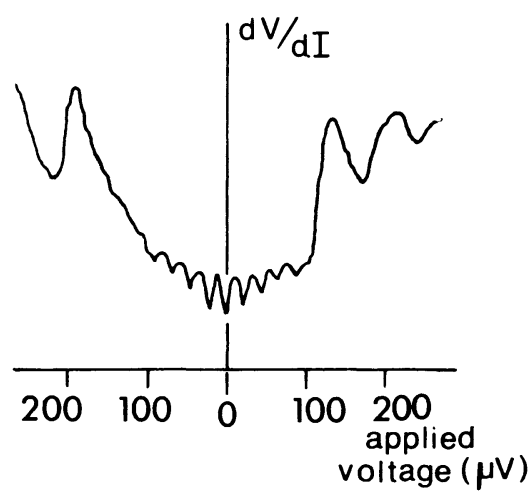

FIG. 12. - Detail of the "single » quantum voltage steps at small bias voltages. The minima are at $20 \mu \mathrm{V}$ intervals.

transition from the uncorrelated to the correlated state can conceivably be thought of as a phase-transition. At higher power levels three junctions seem to be coupled together in this critical region. Certainly synchronization of the whole array is not complete until bias voltages well in excess of $M \hbar \omega / 2$ e are reached.

From the evidence of these curves it seems certain that for particular ranges of microwave power and bias voltage, a perfect two-dimensional point contact array [19] can be driven into synchronization by an external microwave field.

At any given power the synchronizing effect of the microwave field might be expected to weaken for the highest order steps. We appear to have seen the effect of the weakening of the correlation across the array. In figure 13 there is a sudden change of step interval at

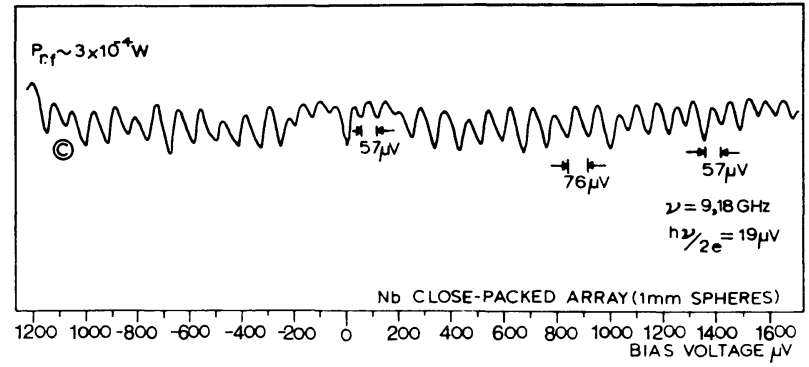

Fig. 13. - Correlated steps for the close-packed array of figure 10 at relatively high rf power levels. Full correlation appears to break down on the high order steps

$$
\text { (at } V_{\text {bias }} \sim 1350 \mu \mathrm{V} \text { ). }
$$

large bias voltages from 76 to $57 \mu \mathrm{V}$. As the microwave power increased so the number of fully correlated steps increased; the "transition » region moved to higher bias voltages. At certain microwave power levels some of the derivative curves for this closepacked array showed transitions

$$
4 \rightarrow 3 \rightarrow 2 \rightarrow 1 \times \hbar \omega / 2 \mathrm{e}
$$

as the bias voltage was increased.

We also tried this synchronization with $4 \mathrm{~mm}$ radiation. The $4 \mathrm{~mm}$ system was coupled to a $3 \mathrm{~cm}$ cryostat 
waveguide by means of a $4 \mathrm{~mm}$ to $3 \mathrm{~cm}$ rectangular cross-section waveguide transition. Obviously the $4 \mathrm{~mm}$ wave was multimoded in the oversized $3 \mathrm{~cm}$ guide. We might therefore expect a rather complicated interaction between the microwave field and the array. This was found to be the case.

Figure 14 shows structure induced in the $\mathrm{d} V / \mathrm{d} I$ versus $V$ curve of a $5 \times 5$ close-packed array of $1 \mathrm{~mm}$ niobium spheres. There is a great wealth of detail at low bias voltages. Presumably each mode can interact with the array separately. However at higher voltages and over a restricted range of microwave power, the derivative curves appear to indicate full correlation across the array with a voltage interval between minima of $600 \mu \mathrm{V}$ (i. e. almost exactly $4 \times \hbar \omega / 2 \mathrm{e}=582 \mu \mathrm{V}$ for $v=70.3 \mathrm{GHz}$ ). It seems reasonable to assume that the strength of the coupling of the array to the $4 \mathrm{~mm}$ radiation field depends on the particular mode involved. For high order steps, where $n$ photons are involved, it may be that the mode best coupled to the array dominates over all others, leading to simplified microwave induced structure in $\mathrm{d} V / \mathrm{d} I$ versus $V$.

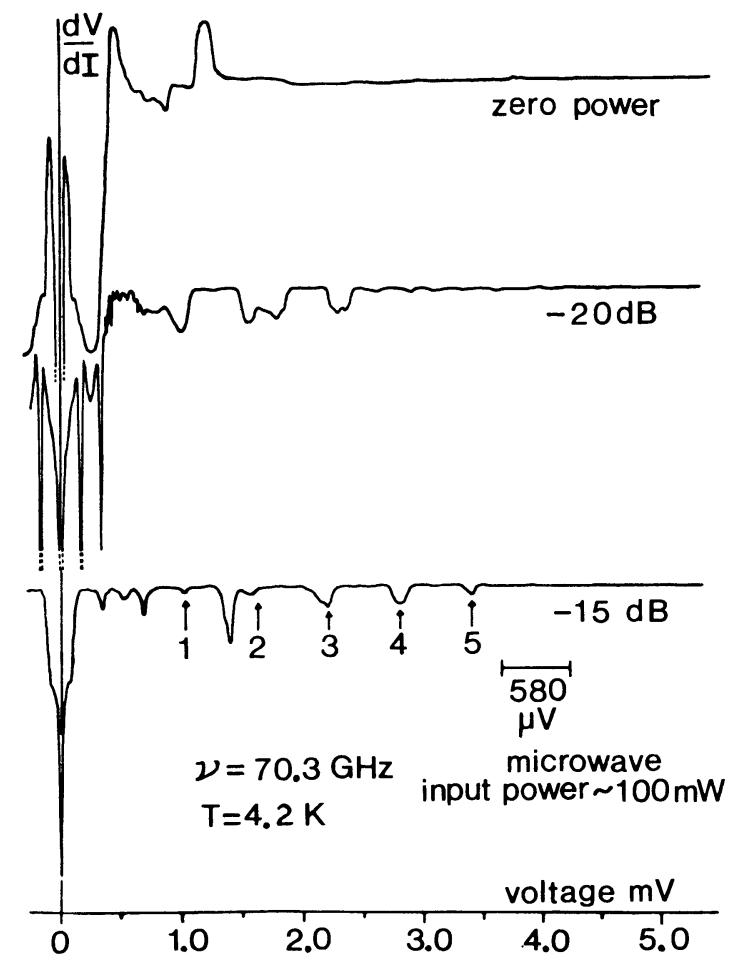

FIG. 14. - Derivative curves for the array of figure 10 at various levels of E-band microwave power. Arrows 1 to 5 on the $-15 \mathrm{~dB}$ curve are separated by $580 \mu \mathrm{V}$. Pronounced minima at voltage intervals of $145 \mu \mathrm{V}(=\hbar \omega / 2 \mathrm{e})$ can be seen close to the voltage origin in the $-20 \mathrm{~dB}$ curve.

5. Microwave detection by point contact arrays. In order to evaluate point contact arrays as broad and narrow and detectors of microwave (X-band) radiation, we either monitored the depression of the $n=0$ zero voltage supercurrent step (after Grimes et al. [31]) or the change in amplitude of a self-induced current step in $I$ versus $V$ (after Richards and Sterling [32]). The former technique provides broad band detection, at least for single point contacts ; the latter, narrow band detection. Conventional modulation and synchronous detection schemes were used throughout [19].

Figures 15 and 16 show the microwave response of a $5 \times 5$ array of $1 \mathrm{~mm} \mathrm{Nb}$ spheres at a temperature of $2.45 \mathrm{~K}$, for various levels of rf power. The junction current is slowly swept from negative to positive, as shown. Peaks $(a)$ in figure 16 correspond to the maximum zero voltage supercurrent in the array. From these data the Noise Equivalent Power (NEP) for broad band detection is found to be

$$
\sim 1 \times 10^{-14} \mathrm{~W} \sqrt{\mathrm{Hz}} \text {. }
$$

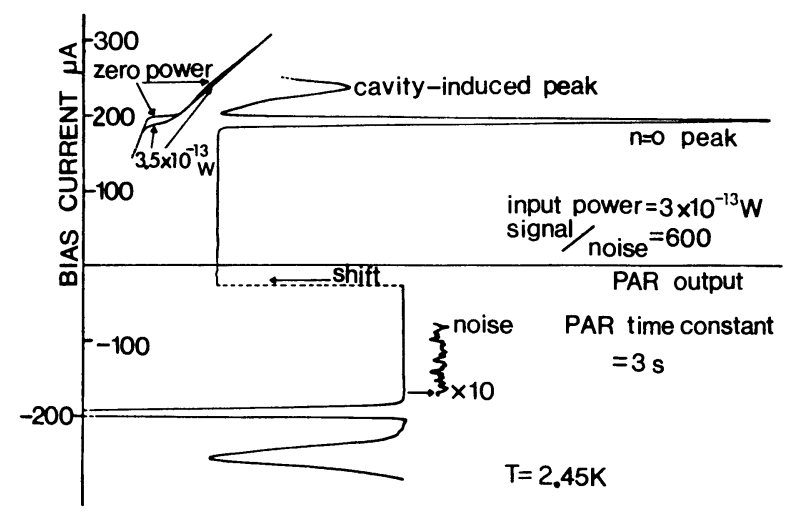

Fig. 15. - X-band response for a $5 \times 5$ square array of $1 \mathrm{~mm}$ $\mathrm{Nb}$ spheres at $T=2.45 \mathrm{~K}$. The relevant section of the $I$ versus $V$ curve is also included. With the electrode contact resistance voltage subtracted the cavity-induced structure occurs at $127.5 \mu \mathrm{V}(15.4 \mathrm{GHz}$ on the single frequency argument).

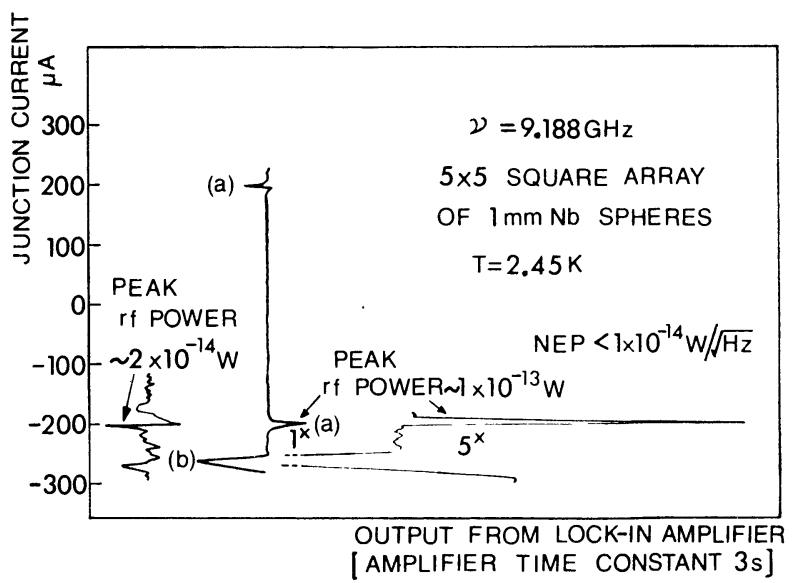

FIG. 16. $-\mathrm{X}$-band response of the figure 15 array at very low microwave power levels. Estimated NEP $\lesssim 1 \times 10^{-14} \mathrm{~W} / \sqrt{\mathrm{Hz}}$.

We have assumed an amplifier band-width of $2 \pi C R$ $(=18.85 \mathrm{~Hz})$ in this calculation. The response of a single junction biased onto the $n=0$ step should be inversely proportional to the square of the microwave frequency [33]. On the single frequency argu- 
ment, at $\lambda=4 \mathrm{~mm}$, the NEP of this array should be $\sim 5 \times 10^{-13} \mathrm{~W} \sqrt{\mathrm{Hz}}$. We ignore any increase in response due to the Riedel-Werthamer peak in the ac current amplitude. This $\lambda=4 \mathrm{~mm}$ estimate is the same as that quoted by Grimes et al. [31] for a single $\mathrm{Nb}-\mathrm{Nb}$ point contact junction.

Rather weak self-induced structure in $I$ versus $V$ was found to produce significant microwave response. For example weak self-induced structure produces the large response marked $(b)$ in figure 16 at microwave power levels $\sim 1 \times 10^{-13} \mathrm{~W}$. On one run with a $5 \times 5$ square array, what we suspected to be cavityinduced structure showed spectacular response to microwaves.

Curve $(a)$ in figure 17 shows the response curve of the array as a function of bias current at a microwave power of $5 \times 10^{-14} \mathrm{~W}$. Unlike the previous response curves this one starts out at zero current. The small peak marked $R_{0}$ corresponds to the $n=0$ step. The current-voltage characteristic is included to make this clear. The large peak marked $R_{\mathrm{c}}$ is the response peak referred to above. At this power level the signal to noise ratio is about 100 . The response curve at the limit of attenuation available $\left(2 \times 10^{-14} \mathrm{~W}\right.$ microwave power ptp) is shown in curve $(b)$. The signal to noise ratio is here about 35 , leading to an estimated (narrow band)

$$
\mathrm{NEP} \sim 2.5 \times 10^{-15} \mathrm{~W} / \sqrt{\mathrm{Hz}}[19] .
$$

For an array in the «superradiant» state the photon density in the surrounding cavity is proportional

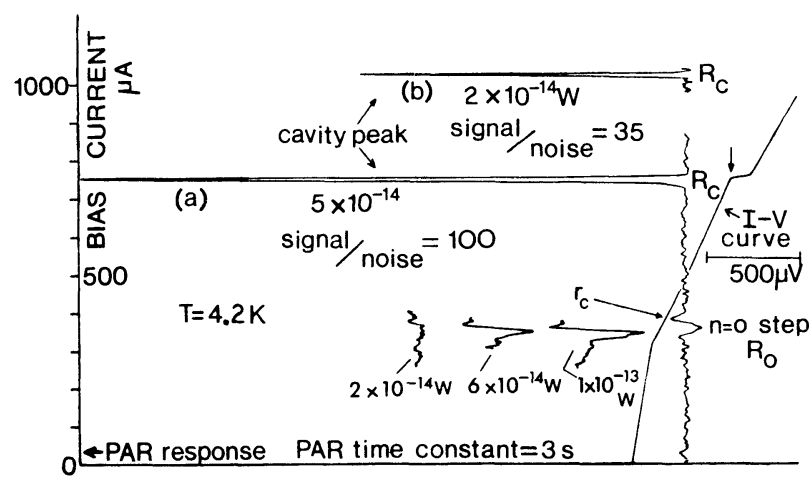

FIG. 17. - X-band response of a $5 \times 5$ array of $1 \mathrm{~mm} \mathrm{Nb}$ spheres as a function of bias current. $R_{0}$ refers to the $n=0$ zero voltage supercurrent response, $r_{\mathrm{c}}$ is the response from weak cavity-induced structure, and $R_{\mathrm{c}}$ is the response from strong self-induced structure. to $M^{2}$, where $M$ is the number of weak links in the array. This common photon field feeds back into the array to produce a current peak (or step) at the cavity voltage $\left(V_{\mathrm{c}}=M \hbar \omega_{\mathrm{c}} / 2 \mathrm{e}\right)$. In narrow band detection it is this step amplitude which is monitored. If this step height goes as $M^{2}$ in arrays which are correlated, the narrow band response will also be proportional to $M^{2}$. It is tempting to identify the large response peak $R_{\mathrm{c}}$ in figure 17 with such a superradiant state.

6. Conclusions. - In the above work we have reviewed some of the experimental evidence for the existence of a single frequency correlated state in planar arrays of point contact Josephson junctions. Some theoretical insight into this phenomenon is provided by Tilley's [8] superradiance argument. The single frequency state apparently is observed, both due to self-synchronization (the Fabry-Pérot experiment) and external synchronization by a microwave field. These experiments were performed on small arrays $(25<M<100)$ where great care was taken to make the arrays regular and uniform. However, for very large arrays (say $M \gtrsim 10^{6}$ ) gross differences between individual weak links should prove tolerable Saxena et al. [12] have observed what appears to be collectively driven behavior in compressed superconducting powders. These powders have been used by Warman et al. [13] as sensitive microwave detectors. In both cases the powder samples consisted of random arrays of point contact junctions.

Correlated array systems obviously hold promise as low power sources in the millimeter and submillimeter regions of the spectrum. On the basis of the Fabry-Pérot experiments described above, coupled arrays with milliwatt power outputs may eventually be realized, utilizing tunnel oxide junctions [24] or microbridges as the radiating elements.

The narrow band detection capabilities of weak link arrays are one area of considerable technological importance. Compared to single weak link detectors, large arrays may show marked improvement in signal to noise performance. Once again, the technical requirement is for rugged, mechanically stable arrays, which obviously precludes the use of point contact junctions. Parametric amplification [1] and microwave harmonic conversion [34] are also fields in which weak link arrays may find useful applications.

\section{References}

[1] Zimmer, H., Appl. Phys. Lett. 10 (1967) 193 ; see also Clorfine, A. S., Appl. Phys. Lett. 4 (1964) 131. [2] Parmenter, R. H., Phys. Rev. 154 (1967) 353 ; 167 (1968) 387.

[3] Anderson, P. W., Prog. Low Temp. Phys. 5 (1967) 40.

[4] Fiory, A. T., Phys. Rev. Lett. 27 (1971) 501.

[5] Clark, T. D. and Tilley, D. R., Phys. Lett. 28A (1968) 62.

[6] McCumber, D. E., J. Appl. Phys. 39 (1968) 297.

[7] Krauss, J. D., Antennas (McGraw-Hill, New York) 1950, p. 222.
[8] Tilley, D. R., Phys. Lett. 33A (1970) 205.

[9] Dayem, A. H. and Grimes, C. C., Appl. Phys. Lett. 9 (1966) 47.

[10] Dicke, R. H., Phys. Rev. 93 (1954) 99.

[11] Schawlow, A. L. and Townes, C. H., Phys. Rev. 112 (1955) 1940.

[12] Saxena, A., Crow, J. E. and Strongin, M., "Coherent Behaviour in Josephson Junction Arrays", Proc. 13th Int. Conf. on Low Temp. Phys. (Boulder, Colorado, 1972), in press. 
[13] Warman, I., Jahn, M. T. and KaO, Y. H., J. Appl. Phys. 42 (1971) 5194.

[14] Shapiro, S., Phys. Rev. Lett. 11 (1963) 80.

[15] Rosenblatt, J., Solid State Commun. 11 (1972) 427.

[16] Cortès, H., Pellan, P. and Rosenblatt, J., Proceedings 12th International Conference on Low Temperature Physics (Keigaku Pub. Co., Tokyo) 1970, p. 487.

[17] Clark, T. D. and Tilley, D. R., Phys. Lett. 29A (1969) 514.

[18] Ambegaokar, V. and Baratoff, A., Phys. Rev. Lett. 10 (1963) 486 ; 11 (1963) 104.

[19] Clark, T. D., Phys. Rev. B 8 (1973) 137.

[20] ATWATER, H. A., Introduction to Microwave Theory (McGraw-Hill, New York) 1962, p. 222.

[21] Clark, T. D., Phys. Lett. 27A (1968) 585.

[22] RePICI, D. J., LeOPOld, L., Gregory, W. D., Behravesh, M. and Thompson, T., Proceedings 1972 Applied Superconductivity Conference (IEEE Pub. No. 72CHO 682-5-TABSC) 1972, p. 701.

[23] Ulrich, B. T. and Kluth, E. O., Proceedings 1972 Applied Superconductivity Conference (IEEE Pub. No. 72CHO 682-5-TABSC) 1972, p. 709.
[24] Finnegan, T. and Wahlsten, S., Appl. Phys. Lett. 21 (1972) 541.

[25] Parker, W. H., Langenberg, D. N., Denenstein, A. and TAYlor, B. N., Phys. Rev. 177 (1969) 639.

[26] Stephen, M. J., Phys. Rev. 186 (1969) 393.

[27] Gregers-Hansen, P. E., Levinsen, M. T. and Fog-PederSEN, G., J. Low Temp. Phys. 7 (1972) 99.

[28] Russer, P., Acta Phys. Austriaca 32 (1970) 373.

[29] Fack, H. and Kose, V., J. Appl. Phys. 42 (1971) 320.

[30] Dayem, A. H. and Weigand, J. J., Phys. Rev. 155 (1967) 419 ;

Wyatt, A. F. G., Dmitriev, V. M., Moore, W. S. and Sheard, F. W., Phys. Rev. Lett. 16 (1966) 1166.

[31] Grimes, C. C., Richards, P. L. and Shapiro, S., J. Appl. Phys. 39 (1968) 3905.

[32] Richards, P. L. and Sterling, S. A., Appl. Phys. Lett. 14 (1969) 394.

[33] Richards, P. L., Physics of III-V Compounds (Academic Press, New York), Vol. 6.

[34] Shapiro, S., J. Appl. Phvs. 38 (1967) 1879. 